\title{
THE CHARLIER B-SERIES
}

\author{
BY \\ R. P. BOAS, JR.
}

1. Introduction. The so-called B-series of Charlier [2] $\left.{ }^{1}\right)$ has been considered in two forms, one for functions defined on the whole real axis ("continuous case") and one for functions defined on the integers ("discrete case"). In either case the formal series is

$$
f(x) \sim \sum_{n=0}^{\infty} a_{n} \Delta^{n} \theta(x)
$$

where

$$
\Delta^{n} \theta(x)=\sum_{k=0}^{n}(-1)^{k} C_{n, k} \theta(x-k)
$$

$\theta(x)$ is given by

$$
\theta(x)=\left\{\begin{array}{cc}
e^{-\lambda} \lambda x / x !, & x \geqq 0, \\
0, & x<0,
\end{array}\right.
$$

in the discrete case (integral $x$ ) and by

$$
\theta(x)=(2 \pi)^{-1} e^{-\lambda} \int_{-\pi}^{\pi} \exp \left\{-i x u+\lambda e^{i u}\right\} d u
$$

in the continuous case; (4) reduces to (3) when $x$ is an integer. There are various formulas for the coefficients $a_{n}$.

The series (1) has an extensive literature; for bibliography see [0], [6], [8], and [9]. However, no attention seems to have been paid to the possibility that some sequence of finite sums of the form

$$
\sum_{k=0}^{N} a_{n}^{(N)} \Delta^{n} \theta(x)
$$

might give a better approximation to a given $f(x)$ than the partial sums of (1) can do. Since in practice only a finite number of terms can be used, this possibility seems to be worth investigating. It turns out that the partial sums of (1) do not in general give as good an approximation as other sums (5); this may explain the fact that (1) has been applied successfully to practical problems although the conditions for its convergence appear rather restrictive.

Presented to the Society, November 27, 1948; received by the editors August 11, 1948.

(1) Numbers in brackets refer to the references at the end of the paper. 
Let us first consider the discrete case and suppose, as is customary, that $f(x)=0$ for $x=-1,-2, \cdots$. The most precise results are those of Schmidt [7], who showed that (1) converges (with the appropriate $a_{n}$ ) if and only if the function

$$
F(z)=\sum_{n=0}^{\infty} f(n) z^{n}
$$

is analytic in $|z|<1$ and in $|z-1|<1$ and is continuous, with all its derivatives, in the closure of this region; in the case of practical interest when $f(n) \geqq 0$, (1) converges if and only if $\lim _{n \rightarrow \infty} f(n) 2^{n} n^{k}=0$ for $k=0,1,2, \cdots$, so that $F(z)$ is analytic in $|z|<2$ and continuous, with all its derivatives, in $|z| \leqq 2$.

However, we shall show in this paper that if $\sum_{n=0}^{\infty}|f(n)|^{2}$ converges, which requires only a little more than the analyticity of $F(z)$ in $|z|<1, f(x)$ can be uniformly approximated (and rather more) by sums (5). A suitable choice of the coefficients $a_{n}^{(N)}$ in (5) is

$$
a_{n}^{(N)}=e^{\lambda}(-1)^{n} \sum_{l=n}^{N} C_{l, n} \sum_{k=0}^{l} \frac{(-\lambda)^{l-k}}{(l-k) !} f(k) ;
$$

these coefficients have the additional property that they make (5) agree exactly with $f(x)$ for $x=0,1,2, \cdots, N$. The usual formula for the coefficients $a_{n}$ in (1) is

$$
\begin{aligned}
a_{n} & =\frac{(-1)^{n}}{n !} \sum_{k=0}^{\infty} f(k) \frac{d^{n}}{d z^{n}}\left(z^{k} e^{\lambda(1-z)}\right)_{z=1} \\
& =\frac{1}{n !} \sum_{k=0}^{\infty} f(k) \sum_{l=0}^{\min (k, n)}(-1) l ! C_{n, l} C_{k, l} \lambda^{n-l} \\
& =\frac{1}{n !} \sum_{l=0}^{n} C_{n, l}(-1)^{l} l ! \lambda^{n-l} \sum_{k=l}^{\infty} f(k) C_{k, l} .
\end{aligned}
$$

If we are to use a finite number of terms of (1) and a finite number of terms of (9) or (10) to obtain approximate coefficients, there is nothing to tell us where to break off the series (9) or (10) to get the best result for a given number of terms of (1). A different formula for the coefficients in (1) can be found by expanding $e^{-\lambda z}$ in a power series before differentiating in (8); then we get

$$
\begin{aligned}
& a_{n}=(-1)^{n} \sum_{k=0}^{\infty} f(k) \sum_{l=\max (k, n)}^{\infty} C_{l, n} e^{\lambda}(-\lambda)^{l-k} / l !, \\
& a_{n}=e^{\lambda}(-1)^{n} \sum_{l=n}^{\infty} C_{l, n} \sum_{k=0}^{l} \frac{(-\lambda)^{l-k}}{(l-k) !} f(k) .
\end{aligned}
$$


Thus, in order to approximate to $f(x)$ by a finite sum (5) of $N+1$ terms, we may break off the formal series (11) for $a_{n}$ at $l=N$ to get $a_{n}^{(N)}$.

It should be noted that the coefficients (7) do not give the closest approximation of $f(x)$ by sums (5) even when they converge, and are thus not, in general, suitable for practical curve-fitting. Some practical aspects of the problem are discussed elsewhere by the author [0].

We now turn to the continuous case. Here most of the results in the literature are negative. Selberg [9] showed that (1) cannot converge uniformly on every finite interval if $f(x) \geqq 0$, and that, unless the Fourier transform of $f(x)$ vanishes outside $(-\pi, \pi)$, the series cannot represent $f(x)$ (with uniform convergence on every finite interval). The special character of the functions representable by (1) in the continuous case is somewhat surprising since J $\phi$ rgensen [5] claims to apply it successfully to practical problems.

We now ask, not when (1) will converge to $f(x)$, but when finite sums of the form (5) will give a good approximation to $f(x)$. As a measure of the closeness of the approximation we shall use the mean square difference,

$$
\int_{-\infty}^{\infty}\left|f(x)-\sum_{n=0}^{N} a_{n}^{(N)} \Delta^{n} \theta(x)\right|^{2} d x .
$$

Let $g(x)$ be the Fourier transform of $f(x)$. We find that, if $f(x) \in L^{2}(-\infty, \infty)$ and $f(x)=0$ when $x$ is a negative integer, (12) can be made arbitrarily close to $(2 \pi)^{-1} \int_{|x|>\pi}|g(x)|^{2} d x$. When $g(x)=0$ almost everywhere in $|x|>\pi$, so that (12) can be made arbitrarily small, a suitable choice of the coefficients $a_{n}^{(N)}$ is $(7)$, so that the coefficients depend only on the values of $f(x)$ for integral $x$ (these values are known to determine $f(x)$ in this case).

If $g(x)=0$ almost everywhere in $|x|>\pi$, the problem of minimizing (12) will be shown to be equivalent to that of minimizing

$$
\sum_{x=0}^{\infty}\left|f(x)-\sum_{n=0}^{N} a_{n}^{(N)} \Delta^{n} \theta(x)\right|^{2} .
$$

This leads us to consider (13) on its own merits. For functions $f(x)$ of integral $x$, the convergence to zero of (13) is a rather strong kind of convergence, since it implies more than the uniform convergence of the sums (5) over $x=0,1,2, \cdots$. Nevertheless, if we suppose only that $\sum_{x=0}^{\infty}|f(x)|^{2}<\infty$, (13) can be made arbitrarily small if the coefficients $a_{n}^{(N)}$ are chosen in accordance with (7).

It is of some interest to consider the possibility that the series (1) converges in the mean, that is, that (12) or (13) approaches zero, with $a_{n}^{(N)}$ independent of $N$. We shall show that this is impossible in the continuous case if $f(x) \geqq 0$ (in agreement with Selberg's result for convergence on every finite interval). For the discrete case, we find that convergence in the mean of (1) implies that the function $F(z)$ of $(6)$ is analytic in $|1-z|<2$; if $f(x) \geqq 0$, $F(z)$ is even analytic in $|z|<3$. 
Selberg [8], [9] studied the generalization of (1) in which (3) and (4) are replaced by

$$
\theta(x)=(2 \pi)^{-1} \int_{-\pi}^{\pi} \phi(u) e^{-i x u} d u
$$

In the rest of this paper we shall consider the more general $\theta(x)$ given by (14); all the special hypotheses to be placed on $\theta(x)$ will be satisfied by the original function (4).

We shall also, in most of the paper, eliminate the need for supposing that $f(x)=0$ for $x=-1,-2, \cdots$, by generalizing (1) to include, in addition to the differences $\Delta^{n} \theta(x)$ defined in (2), the differences

$$
\nabla^{n} \theta(x)=\sum_{k=0}^{n}(-1)^{k} C_{k, n} \theta(x+k) .
$$

This makes the theory more symmetrical.

2. The continuous case. It is sometimes convenient to think of (14) as

$$
\theta(x)=(2 \pi)^{-1} \int_{-\infty}^{\infty} \phi(u) e^{-i x u} d u,
$$

where $\phi(u)$ belongs to $L^{2}$ and we define $\phi(u)$ to be 0 outside $(-\pi, \pi)$. We have for $n=0,1,2, \cdots$,

$$
\begin{aligned}
& \Delta^{n} \theta(x)=(2 \pi)^{-1} \int_{-\pi}^{\pi} \phi(t)\left(1-e^{i t}\right)^{n} e^{-i x t} d t, \\
& \nabla^{n} \theta(x)=(2 \pi)^{-1} \int_{-\pi}^{\pi} \phi(t)\left(1-e^{-i t}\right)^{n} e^{-i x t} d t .
\end{aligned}
$$

Let $f(x)$ belong to $L^{2}(-\infty, \infty)$. We consider the problem of finding coefficients $a_{k}$ and $b_{k}$ (in general depending on $n$, although in order to simplify the notation we do not indicate this dependence) which will minimize the expression

$$
D_{n}=\int_{-\infty}^{\infty}\left|f(x)-\sum_{k=0}^{n} a_{k} \Delta^{k} \theta(x)-\sum_{k=1}^{n} b_{k} \nabla^{k} \theta(x)\right|^{2} d x
$$

Let

$$
g(x)=\int_{-\infty}^{\infty} e^{i x t} f(t) d t
$$

where, as usual in the theory of Fourier integrals, the integral is taken as a limit in the mean of $\int_{-T}^{T}$ as $T \rightarrow \infty$. By Parseval's formula for Fourier transforms, we have 


$$
\begin{aligned}
2 \pi D_{n}= & \int_{-\infty}^{\infty}\left|g(t)-\phi(t) \sum_{k=0}^{n} a_{k}\left(1-e^{i t}\right)^{k}-\phi(t) \sum_{k=1}^{n} b_{k}\left(1-e^{-i t}\right)^{k}\right|^{2} d t \\
= & \int_{|t|>\pi}|g(t)|^{2} d t+\int_{-\pi}^{\pi} \mid g(t)-\phi(t) \sum_{k=0}^{n} a_{k}\left(1-e^{i t}\right)^{k} \\
& -\left.\phi(t) \sum_{k=1}^{n} b_{k}\left(1-e^{-i t}\right)^{k}\right|^{2} d t
\end{aligned}
$$

From (21) we see that

$$
2 \pi D_{n} \geqq G \equiv \int_{|t|>\pi}|g(t)|^{2} d t,
$$

so that the minimum of $D_{n}$ is certainly positive unless $g(t)=0$ almost everywhere outside $(-\pi, \pi)$.

Let us now suppose that $0<m \leqq|\phi(t)| \leqq M<\infty$ almost everywhere in $(-\pi, \pi)$. Let $1 / \phi(t)$ and $g(t)$ have the Fourier series

$$
\begin{aligned}
1 / \phi(t) & \sim \sum_{n=-\infty}^{\infty} p_{n} e^{i n t}, \\
g(t) & \sim \sum_{n=-\infty}^{\infty} \gamma_{n} e^{i n t} ;
\end{aligned}
$$

then the Fourier series of $g(t) / \phi(t)$ is

$$
g(t) / \phi(t) \sim \sum_{n=-\infty}^{\infty} c_{n} e^{i n t}
$$

where

$$
c_{n}=\sum_{k=-\infty}^{\infty} p_{k} \gamma_{n-k}
$$

Now the Fourier series of a function of $L^{2}$ converges in the mean, and so if $\epsilon>0$ we can find an $n$ such that $\int_{-\pi}^{\pi}\left|g(t) / \phi(t)-\sum_{k=-n}^{n} c_{k} e^{i k t}\right|^{2} d t<\epsilon$. We then can determine $a_{k}(k=0,1, \cdots, n)$ and $b_{k}(k=1, \cdots, n)$ so that

$$
\sum_{k=0}^{n} a_{k}\left(1-e^{i t}\right)^{k}+\sum_{k=1}^{n} b_{k}\left(1-e^{-i t}\right)^{k}=\sum_{k=0}^{n} c_{k} e^{i k t}+\sum_{k=1}^{n} c_{-k} e^{-i k t},
$$

that is,

$$
a_{k}=(-1)^{k} \sum_{l=k}^{n} C_{l, k} c_{l}, \quad b_{k}=(-1)^{k} \sum_{l=k}^{n} C_{l, k} C_{-l} .
$$

Since 


$$
2 \pi D_{n}=G+\int_{-\pi}^{\pi}|\phi(t)|^{2}\left|g(t) / \phi(t)-\sum_{k=0}^{n} a_{k}\left(1-e^{i t}\right)^{k}-\sum_{k=1}^{n} b_{k}\left(1-e^{-i t}\right)^{k}\right|^{2} d t,
$$

and $m \leqq|\phi(t)| \leqq M$ almost everywhere in $(-\pi, \pi)$, it follows that $2 \pi D_{n} \leqq G$ $+M^{2} \epsilon$, where $G$ is defined in (22). Thus $D_{n}$ can be made arbitrarily small by suitable choice of $a_{k}$ and $b_{k}$ if $G=0$, and only if $G=0$; also, we can have $b_{k}=0$ $(1 \leqq k \leqq n)$ if and only if $c_{-l}=0$ for $l=1,2, \cdots$.

We collect our results so far in a theorem.

TheOREM 1. Let $f(x) \in L^{2}(-\infty, \infty)$ and let $g(t)$ be its Fourier transform (20). Let $0<m \leqq|\phi(x)| \leqq M<\infty$ almost everywhere in $(-\pi, \pi)$. Then

$$
\min \int_{-\infty}^{\infty}\left|f(x)-\sum_{k=0}^{n} a_{k}^{(n)} \Delta^{k} \theta(x)-\sum_{k=1}^{n} b_{k}^{(n)} \nabla^{k} \theta(x)\right|^{2} d x=0
$$

if and only if $\mathrm{g}(t)=0$ almost everywhere outside $(-\pi, \pi)$; and

$$
\min \int_{-\infty}^{\infty}\left|f(x)-\sum_{k=0}^{n} a_{k}^{(n)} \Delta^{k} \theta(x)\right|^{2} d x=0
$$

if and only if, in addition, all the Fourier coefficients of negative index for $g(x) / \phi(x)$ are zero. The minimum in (28) and (29) is taken with respect to all sequences $\left\{a_{k}^{(n)}\right\},\left\{b_{k}^{(n)}\right\}$.

Now let us suppose that $g(t)=0$ almost everywhere outside $(-\pi, \pi)$; then in the Fourier series (24) for $g(t)$ we have $\gamma_{n}=f(n)$. If we also have $f(n)$ $=p_{n}=0$ for $n<0$ in the series (23) for $1 / \phi(t)$, we have the following expression for the Fourier coefficients (26) of $g(t) / \phi(t)$ :

$$
\begin{aligned}
c_{n} & =\sum_{k=0}^{n} p_{k} f(n-k), & & n \geqq 0, \\
c_{n} & =0, & & n<0 .
\end{aligned}
$$

Hence we have the following theorem.

ThEOREM 2. Let $f(x) \in L^{2}(-\infty, \infty)$ and let its Fourier transform $g(t)$ vanish almost everywhere for $|t|>\pi$. Let $0<m \leqq|\phi(x)| \leqq M<\infty$ almost everywhere in $(-\pi, \pi)$. Let $f(n)=0$ for $n=-1,-2, \cdots$, and let all coefficients of negative index in the Fourier series (23) for $1 / \phi(t)$ be zero. Then

$$
\lim _{n \rightarrow \infty} \int_{-\infty}^{\infty}\left|f(x)-\sum_{k=0}^{n} a_{k}^{(n)} \Delta^{k} \theta(x)\right|^{2} d x=0,
$$

where

$$
a_{k}^{(n)}=(-1)^{k} \sum_{l=k}^{n} C_{l, k} \sum_{j=0}^{l} p_{j} f(l-j)
$$


The coefficients (32) were determined so that $\sum_{k=0}^{n} a_{k}^{(n)}\left(1-e^{i t}\right)^{k}$ is identical with the terms in $1, e^{i t}, \cdots, e^{i n t}$ of the Fourier series of $g(t) / \phi(t)$, so that the corresponding Fourier coefficients of $g(t) / \phi(t)-\sum_{k=0}^{n} a_{k}^{(n)}\left(1-e^{i t}\right)^{k}$ are zero. If all the Fourier coefficients of negative index of $\phi(t)$ are zero (as they are for Charlier's function (4)) this means that the coefficients of $1, e^{i t}, \cdots$, $e^{i n t}$ are all zero for $g(t)-\phi(t) \sum_{k=0}^{n} a_{k}^{(n)}\left(1-e^{i t}\right)^{k}$; but these coefficients are precisely $f(x)-\sum_{k=0}^{n} a_{k}^{(n)} \Delta^{k} \theta(x), x=0,1,2, \cdots, n$. Thus in case all the Fourier coefficients of negative index of $\phi(t)$ as well as of $1 / \phi(t)$ are zero, the sums in (31) agree exactly with $f(x)$ for $x=0,1,2, \cdots, n$.

In particular, the hypotheses on $\phi(x)$ are satisfied by $\phi(x)=\exp \left\{\lambda\left(e^{i x}-1\right)\right\}$, the original Charlier function. We have, in fact, $p_{k}=e^{\lambda}(-\lambda)^{k} / k$ ! for $k \geqq 0$, $p_{k}=0$ for $k<0$. Thus in this case we have formula (7) for $a_{n}^{(N)}$, and exact agreement with $f(x)$ for $k=0,1,2, \cdots, N$.

3. The discrete case. Here $x$ takes only integral values. We have

$$
\theta(x)=(2 \pi)^{-1} \int_{-\pi}^{\pi} \phi(u) e^{-i x u} d u
$$

and

$$
\phi(u) \sim \sum_{x=-\infty}^{\infty} \theta(x) e^{i x u} .
$$

We suppose as before that $0<m \leqq|\phi(u)| \leqq M<\infty$. Let us assume that $\sum_{-\infty}^{\infty}|f(x)|^{2}$ converges; then we can define

$$
g(t)=\sum_{x=-\infty}^{\infty} e^{i x t} f(x)
$$

with the series converging in mean square, and seek to minimize

$$
D_{n}=\sum_{x=-\infty}^{\infty}\left|f(x)-\sum_{k=0}^{n} a_{k}^{(n)} \Delta^{k} \theta(x)-\sum_{k=1}^{n} b_{k}^{(n)} \nabla^{k} \theta(x)\right|^{2},
$$

where $\Delta^{k} \theta(x)$ and $\nabla^{k} \theta(x)$ are defined in (17) and (18). Now

$$
f(x)=(2 \pi)^{-1} \int_{-\pi}^{\pi} g(t) e^{-i x t} d t, \quad x=0, \pm 1, \pm 2, \cdots ;
$$

hence

$$
2 \pi D_{n}=\int_{-\pi}^{\pi}\left|g(t)-\phi(t) \sum_{k=0}^{n} a_{k}^{(n)}\left(1-e^{i t}\right)^{k}-\phi(t) \sum_{k=1}^{n} b_{k}^{(n)}\left(1-e^{-i t}\right)^{k}\right|^{2} d t .
$$

This is precisely (21) under the assumption that $g(t)=0$ almost everywhere outside $(-\pi, \pi)$. Moreover, if $f(x)$ is defined for nonintegral $x$ by (37), it satisfies the hypotheses of $\$ 2$. Hence we have the following theorem. 
TheOREM 3. The problem of minimizing $D_{n}$ defined by (36) for an $f(x)$ defined for integral $x$ with $\sum_{-\infty}^{\infty}|f(x)|^{2}$ convergent is equivalent to the problem of minimizing $D_{n}$ defined by the equation (19) for an $f(x)$ defined for all real $x$ with $\int_{-\infty}^{\infty}|f(x)|^{2} d x$ convergent and with the Fourier transform of $f(x)$ vanishing outside $(-\pi, \pi)$.

We can now translate Theorems 1 and 2 into theorems for functions of the integral-valued variable $x$.

Theorem 4. Let $\sum_{-\infty}^{\infty}|f(x)|^{2}$ converge and let $g(t)$ be defined by (35). Let $0<m \leqq|\phi(x)| \leqq M<\infty$ almost everywhere in $(-\pi, \pi)$. Then

$$
\min \sum_{-\infty}^{\infty}\left|f(x)-\sum_{k=0}^{n} a_{k}^{(n)} \Delta^{k} \theta(x)-\sum_{k=1}^{n} b_{k}^{(n)} \nabla^{k} \theta(x)\right|^{2}=0 ;
$$

and furthermore

$$
\min \sum_{-\infty}^{\infty}\left|f(x)-\sum_{k=0}^{n} a_{k}^{(n)} \Delta^{k} \theta(x)\right|^{2}=0
$$

if and only if all the Fourier coefficients of negative index for $g(x) / \phi(x)$ are zero. The last condition is satisfied if $f(x)=0$ for $x=-1,-2, \cdots$ and all Fourier coefficients of negative index in the Fourier series (23) for $1 / \phi(t)$ are zero. In this case the coefficients $a_{k}^{(n)}$ are given by (32). If in addition all Fourier coefficients of negative index of $\phi(t)$ are zero, the sums in (40) agree with $f(x)$ for $x=0,1,2$, ..., n.

4. Representation by a series. In general we may expect to get a closer approximation to $f(x)$ by using linear combinations of $\Delta^{k} \theta(x)$ other than the partial sums of the formal series expansion of $f(x)$. However, it is of interest to ask when the series will furnish a good approximation, in the sense that

$$
\lim _{n \rightarrow \infty} \int_{-\infty}^{\infty}\left|f(x)-\sum_{k=0}^{n} a_{k} \Delta^{k} \theta(x)\right|^{2} d x=0
$$

(continuous case) or

$$
\lim _{n \rightarrow \infty} \sum_{x=-\infty}^{\infty}\left|f(x)-\sum_{k=0}^{n} a_{k} \Delta^{k} \theta(x)\right|^{2}=0
$$

(discrete case), where the $a_{k}$ do not depend on $n$. We shall consider only the particular case (which includes the original $\theta(x)$ of (3) and (4)) where (in the continuous case) $g(t)=0$ almost everywhere outside $(-\pi, \pi)$, the Fourier coefficients of negative index of $1 / \phi(t)$ are zero, and $\theta(x)=f(x)=0$ for $x=-1$, $-2, \cdots$ Then from our results so far it follows that (41) and (42) are equivalent to 


$$
\lim _{n \rightarrow \infty} \int_{-\pi}^{\pi}\left|g(t)-\phi(t) \sum_{k=0}^{n} a_{k}\left(1-e^{i t}\right){ }^{k}\right|^{2} d t=0 .
$$

If (43) holds, the series $\sum_{k=0}^{\infty} a_{k} \phi(t)\left(1-e^{i t}\right)^{k}$ converges in mean square; hence its general term converges to zero in mean square. That is,

$$
\lim _{n \rightarrow \infty}\left|a_{n}\right|^{2} \int_{-\pi}^{\pi}|\phi(t)|^{2}\left|1-e^{i t}\right|^{2 n} d t=0,
$$

or

$$
\lim _{n \rightarrow \infty} 2^{2 n}\left|a_{n}\right|^{2} \int_{-\pi}^{\pi}|\phi(t)|^{2} \sin ^{2 n}(t / 2) d t=0 .
$$

Under our hypotheses, $|\phi(t)| \geqq m>0$ and so

$$
\begin{aligned}
2^{2 n}\left|a_{n}\right|^{2} \int_{-\pi}^{\pi}|\phi(t)|^{2} \sin ^{2 n}(t / 2) d t & \geqq 2^{2 n}\left|a_{n}\right|^{2} m^{2} \int_{-\pi}^{\pi} \sin ^{2 n}(t / 2) d t \\
& =2^{2 n+1}\left|a_{n}\right|^{2} m^{2} \pi^{1 / 2} \Gamma(n+1 / 2) / n ! ;
\end{aligned}
$$

consequently

$$
a_{n}=o\left(2^{-n} n^{1 / 4}\right)
$$

and so $\sum_{n=0}^{\infty} a_{n}\left(1-e^{i t}\right)^{n}$ converges for $|t|<\pi$, since $\left|1-e^{i t}\right|<2$ if $|t|<\pi$. Hence, almost everywhere in $(-\pi, \pi)$,

$$
g(t)=\phi(t) \sum_{k=0}^{\infty} a_{k}\left(1-e^{i t}\right)^{k} .
$$

Now we also have

$$
g(t) / \phi(t)=\sum_{n=0}^{\infty} c_{n} e^{i n t}, \quad|t|<\pi,
$$

where the $c_{n}$ are given by (26). Let us consider the following functions in $|z|<1$ :

$$
F(z)=\sum_{n=0}^{\infty} f(n) z^{n}, \quad \Psi(z)=\sum_{n=0}^{\infty} p_{n} z^{n}, \quad P(z)=\sum_{n=0}^{\infty} c_{n} z^{n}=F(z) \Psi(z) ;
$$

here $p_{n}$ and $c_{n}$ are the coefficients in (23) and (26). Since $\sum\left|c_{n}\right|^{2}$ converges, $P(z)$ has, by a well known theorem (see, for example $[10$, p. 244]), radial boundary values $P\left(e^{i t}\right)$ on $|z|=1$, equal (according to (45)) to $g(t) / \phi(t)$ for almost all $t$. Now consider

$$
H(z)=\sum_{n=0}^{\infty} a_{n}(1-z)^{n},
$$


where the $a_{n}$ are the coefficients in (43). By (44), $H(z)$ is analytic in $|1-z|<2$ and by $(45) H\left(e^{i t}\right)=g(t) / \phi(t)$ almost everywhere in $-\pi \leqq t \leqq \pi$, since $|z|=1$ is (except for $z=-1$ ) inside the circle where $H(z)$ is analytic. Thus $H(z)$ provides an analytic extension of $P(z)$ into $|1-z|<2$ and so $F(z)$ is analytic in $|1-z|<2$ provided that $1 / \Psi(z)$ is. Now let

$$
\Phi(z)=\sum_{n=0}^{\infty} \theta(n) z^{n}
$$

since $\sum_{0}^{\infty} \theta(n) e^{i n t}$ is the Fourier series of $\phi(t)$, and $\sum_{0}^{\infty} p_{n} e^{i n t}$ is the Fourier series of $1 / \phi(t)$, we have $\Phi(z)=1 / \Psi(z)$ on $|z|=1$ and so wherever either function is analytic. Hence $F(z)$ is analytic in $|1-z|<2$ if $\Phi(z)$ is. We thus have the following theorem.

ThEOREM 5. If (i) $\theta(x)=f(x)=0$ for $x=-1,-2, \cdots$; (ii) (in the continuous case) $g(t)=0$ almost everywhere outside $(-\pi, \pi)$; (iii) all the Fourier coefficients of negative index of $1 / \phi(t)$ are zero; (iv) $\Phi(z)$ defined by (48) is analytic in $|1-z|<2$; and (v) (42) holds, then $F(z)$ is analytic in $|1-z|<2$.

In particular, the original $\theta(x)$ of (3) and (4) satisfies the hypotheses.

Conversely, if $F(z)$ and $\Psi(z)=1 / \Phi(z)$ are analytic in $|1-z|<2$, and $H(z)=F(z) \Psi(z)$, let $H(z)=\sum_{n=0}^{\infty} a_{n}(1-z)^{n}$ for $|1-z|<2$; if $\sum_{0}^{\infty} a_{n}\left(1-e^{i t}\right)^{n}$ converges in mean square on $|z|=1$, under our hypotheses on $\phi(t)$ we have (43). This will in particular be true if $H(z)$ is analytic in $|1-z| \leqq 2$. Hence we have the following partial converse of Theorem 5 .

THEOREM 6. If hypotheses (i), (ii), (iii) of Theorem 5 are satisfied and if $F(z)$ and $1 / \Phi(z)$ are analytic in $|1-z| \leqq 2$, then (43) is true. In particular, in the discrele case the series (1) converges uniformly. The coefficients $a_{k}$ in (43) are obtained by taking $N=\infty$ in (7).

Uniform convergence in the discrete case was established by Selberg [8] if $1 / \Phi(z)$ is analytic in $|1-z| \leqq 2$ and $\sum f(n) 3^{n}$ converges (so that $F(z)$ is analytic in $|z|<3$ ); Selberg assumes somewhat less about $\theta(x)$ than we do, however.

We next consider the effect of having $f(n) \geqq 0$. In this case $F(z)$ is a power series with non-negative coefficients and consequently has a singular point at the real positive point on its circle of convergence; see, for example, $[10$, p. 214]. But if the hypotheses of Theorem 5 are satisfied, $F(z)$ has no singular point in $-1<x<3$, and so must be analytic at least in $|z|<3$. Hence we have the following theorem.

THEOREM 7. If, in addition to the hypotheses of Theorem $5, f(n) \geqq 0$, then $F(z)$ is analytic in $|z|<3$.

In the continuous case, if $f(x) \not \equiv 0, f(x) \geqq 0$ for $x=0,1,2, \cdots$, and $f(x)=0$ for $x=-1,-2, \cdots$, and if $(41)$ holds, we know that $g(t)=0$ almost 
everywhere outside $(-\pi, \pi)$. It follows that $g(t)$ cannot be analytic at $t=0$. In fact, it is well known [3, p. 90] that, if $f(x) \geqq 0$, and $g^{(n)}(0)$ exists for $n=1,2, \cdots$, then $\int_{-\infty}^{\infty}|x| n f(x) d x$ exists for $n=1,2, \cdots$, and so $g^{(n)}(t)$ exists for $n=1,2, \cdots$ and all $t$. Furthermore, $\left|g^{(2 n)}(t)\right| \leqq\left|g^{(2 n)}(0)\right|$ $=(2 \pi)^{-1} \int_{-\infty}^{\infty} x^{2 n} f(x) d x=\mu_{2 n}$, say. Therefore $[1, \mathrm{p} .12]\left|g^{(2 n+1)}(t)\right| \leqq\left(2 \mu_{2 n} \mu_{2 n+2}\right)^{1 / 2}$. It follows that $g(t)$ is analytic at every $t$ if it is analytic at $t=0$.

On the other hand, we have $H(z)$ analytic, in particular, at $|z|=1$, and $H\left(e^{i t}\right)=g(t) / \phi(t)$ in a (real) neighborhood of $t=0$. If now $t$ is regarded as a complex variable, $H\left(e^{i t}\right)$ is analytic in a (complex) neighborhood of $t=0$ and so $g(t) / \phi(t)$ is analytic at $t=0$. Since $g(t)$ is not analytic at $t=0, \phi(t)$ cannot be analytic there either. We have thus established the following theorem.

Theorem 8. If $f(x) \not \equiv 0, f(x) \geqq 0$ for $x=0,1,2, \cdots, f(x)=0$ for $x=-1$, $-2, \cdots$, and $\phi(t)$ is analytic at $t=0$, then (41) cannot hold.

This was shown by Selberg [9] under somewhat different hypotheses on $\phi(t)$, and for uniform convergence on every finite interval instead of (41), but under the more restrictive hypothesis that $f(x) \geqq 0$ for all real $x$ instead of for all integral $x$.

\section{REFERENCES}

0. R. P. Boas, Jr., The representation of probability distributions by Charlier series, Ann. Math. Statist. vol. 20 (1949) pp. 376-392.

1. T. Carleman, Les fonctions quasi analytiques, Paris, 1926.

2. C. V. L. Charlier, Über die Darstellung willkürlicher Funktionen, Arkiv för Matematik, Astronomi och Fysik vol. 2 (1906) no. 20.

3. H. Cramér, Mathematical methods of statistics, Princeton, 1946.

4. N. R. Jфrgensen, Note sur la fonction de répartition de type $B$ de M. Charlier, Arkiv för Matematik, Astronomi och Fysik vol. 10 (1915) no. 15.

5. - Undersфgelser over Frequensflader og Korrelation, Copenhagen thesis, 1916.

6. S. Kullback, On the Charlier type B series, Ann. Math. Statist. vol. 18 (1947) pp. 574581 ; vol. 19 (1948) p. 427.

7. E. Schmidt, Über die Charlier-Jordansche Entwicklung einer willkïrlichen Funktion nach der Poissonschen Funktionen und ihren Ableitungen, Zeitschrif t für Angewand te Mathematik und Mechanik vol. 13 (1933) pp. 139-142.

8. H. L. Selberg, Über die Darstellung willkürlicher Funktionen durch Charliersche Differenzreihen, Skandinavisk Aktuarietidskrift vol. 25 (1942) pp. 228-246.

9. — , Über die Darstellung der Dichtefunktion einer Verteilung durch eine Charliersche $B$-Reihe, Archiv for Mathematik og Naturvidenskab vol. 46 (1943) pp. 127-138.

10. E. C. Titchmarsh, The theory of functions, Oxford, 1932.

Brown UNIVERSITY,

Providence, R. I.

Massachusetts Institute of Technology, Cambrinff, Mass. 